\title{
ON THE WEAK CONVERGENCE OF SIMILAR PROBABILITY LAWS
}

RAMACHANDRAMURTY PONNAPALLI*

The concept of types of random variables can be extended to random vectors in several ways. It is shown in this paper that with some natural extensions of this concept, convergence of laws is in reality convergence of types as in the case of random variables.

Definition 1 . We say two random variables $X$ and $Y$ (or their probability laws) are of the same (positive) type if $\exists b>0$ and $a$ such that $\mathcal{L}(Y)=\mathcal{L}(a+b X)$.

Definition 2. We say a random variable $X$ (or its law) is degenerate if $\exists c$ such that $P(X=c)=1$.

Definition 3. We say a distribution function $F(x)$ is defective if $F(+\infty)-F(-\infty)<1$, or equivalently, if $F(+\infty)<1$, or $F(-\infty)$ $>0$ or both.

In this paper we are primarily concerned with distribution functions of random variables which are necessarily nondefective.

Definition 4. We say $U_{n}$ converges weakly to $U\left(U_{n} \stackrel{w}{\rightarrow} U\right)$ or $\mathcal{L}\left(U_{n}\right) \rightarrow \mathcal{L}(U)$ if for every continuity point $z$ of $F(u)=$ $P(U \leqq u), P\left(U_{n} \leqq z\right)=F_{n}(z) \rightarrow F(z)$.

This definition holds both for random variables and random vectors.

We restate the familiar result about the weak convergence of types of random variables as follows. If, for sufficiently large $n, X_{n}$ and $Y_{n}$ are of the same type and $X_{n} \stackrel{w}{\rightarrow}$ nondegenerate $X$ and $Y_{n} \stackrel{w}{\rightarrow}$ nondegenerate $Y$, then $X$ and $Y$ are of the same type [1, Theorem $1, p$. 40]. It is this result that justifies the usage that a sequence of random variables $Z_{n}$ has, asymptotically, a normal distribution (without specifying the norming constants). Since all (univariate) nondegenerate normal distributions are of the same type it follows that for any sequence of constants $a_{n}$ and $b_{n}>0$ the limit law of $a_{n}+b_{n} Z_{n}$ is also normal possibly with different mean and variance if it is nondefective and nondegenerate.

Received by the editors on May 28, 1974, and in revised form on December 4, 1974.

* Supported in part by NRC of Canada Grant A-7575. 
The author introduced the following concepts of similar probability laws of random vectors in [3].

Definition 5. We say two random vectors $U^{\prime}=\left(U_{1}, U_{2}, \cdots, U_{p}\right)$ and $V^{\prime}=\left(V_{1}, V_{2}, \cdots, V_{p}\right)$ or their probability laws are similar if $\exists$ a nonrandom vector $\boldsymbol{A}^{\prime}=\left(a_{1}, a_{2}, \cdots, a_{p}\right)$ and a nonrandom nonsingular $p \times p$ matrix $B=\left(b_{i j}\right)$ such that $\mathcal{L}(V)=\mathcal{L}(A+B U)$. We then write $U \sim V$. They are said to be strongly similar if $\exists$ a scalar $b \neq 0$ such that $\mathcal{L}(\boldsymbol{V})=\mathcal{L}(\boldsymbol{A}+\boldsymbol{b} \boldsymbol{U})$. We write $\boldsymbol{U} \stackrel{s}{\sim}$ if they are strongly similar.

Definition 6. We say a random vector $\boldsymbol{U}$ (or its law) is nondegenerate if there is no hyperplane in $R_{p}$ containing the total mass of the probability distribution of $U$; that is, if there exists no nonrandom $\ell^{\prime}=\left(\ell_{1}, \ell_{2}, \cdots, \ell_{p}\right) \neq(0,0, \cdots, 0)$ such that $P\left(\ell_{1} U_{1}+\ell_{2} U_{2}+\cdots\right.$ $\left.+\ell_{p} U_{p}=\ell\right)=1$ for some scalar $\ell$.

Some authors use 'nonsingular' instead of 'nondegenerate'; the latter is used here to avoid possible confusion with nonsingularity of matrices.

It is clear that $U$ is nondegenerate if and only if $\left(U_{i_{1}}, U_{i_{2}}, \cdots, U_{i_{t}}\right)$ is nondegenerate for all distinct $i_{1}, i_{2}, \cdots, i_{t}$ such that $1 \leqq i_{j} \leqq p, j=$ $1,2, \cdots, t$.

LEMMA 1 . If $P\left(X_{n} \leqq x\right)=F_{n}(x) \stackrel{w}{\rightarrow}$ nondefective $F(x)$ then, (a) $\left|a_{n}\right| \rightarrow \infty \Longrightarrow\left(X_{n} / a_{n}\right) \stackrel{P}{\rightarrow} 0$, (b) $a_{n} \rightarrow \pm \infty \Longrightarrow X_{n}+a_{n}$ converges weakly to a defective probability distribution.

Proof. Given $\epsilon>0$, let $A(\epsilon)$ be a continuity point of $F$ such that $F(A)>1-\epsilon / 4$. Since $F_{n}(A) \rightarrow F(A) \exists L(\epsilon)$ such that $n>L(\epsilon) \Rightarrow$ $\left|F_{n}(A)-F(A)\right|<\epsilon / 2$. Now for $n>L(\epsilon)$ and $x>A(\epsilon)$

$$
\begin{aligned}
&\left|F_{n}(x)-F(x)\right|=\left|\left\{1-F_{n}(x)\right\}-\{1-F(x)\}\right| \leqq 1-F_{n}(x)+1-F(x) \\
& \leqq \\
& \leqq \leqq \\
& \frac{\epsilon}{2}+2 \cdot \frac{\epsilon}{4}=\epsilon .
\end{aligned}
$$

Similarly $\exists M(\epsilon)$ and $B(\epsilon)$ such that $F(B(\epsilon))<\epsilon / 4, x<B(\epsilon)$, and $n>M(\epsilon) \Longrightarrow\left|F_{n}(x)-F(x)\right|<\epsilon$. If we let $N(\epsilon)=\max (L(\epsilon), M(\epsilon))$, then $n>N(\epsilon), x<B(\epsilon)$ or $x>A(\epsilon) \Rightarrow\left|F_{n}(x)-F(x)\right|<\epsilon$. Now given $\delta>0, \exists P(\delta, \epsilon)$ such that $n>P(\delta, \epsilon) \Longrightarrow\left|a_{n}\right| \delta>A(\epsilon)$ and $-\left|a_{n}\right| \delta<$ $B(\epsilon)$. For such $n$ we have

$$
P\left(\left|\frac{X_{n}}{a_{n}}\right|<\delta\right)=F_{n}\left(\left|a_{n}\right| \delta\right)-F_{n}\left(-\left|a_{n}\right| \delta\right)
$$




$$
\begin{aligned}
& \geqq F\left(\left|a_{n}\right| \delta\right)-\epsilon-\left\{F\left(-\left|a_{n}\right| \delta\right)+\epsilon\right\} \\
& =F\left(\left|a_{n}\right| \delta\right)-F\left(-\left|a_{n}\right| \delta\right)-2 \epsilon \\
& \geqq 1-\frac{\epsilon}{4}-\frac{\epsilon}{4}-2 \epsilon=1-\frac{5 \epsilon}{2} .
\end{aligned}
$$

Since $\epsilon$ is arbitrary it follows that $P\left(\left|X_{n}\right| a_{n} \mid<\delta\right) \rightarrow 1$ as $n \rightarrow \infty$ for all $\delta>0$. QED

Part (b) can be proved similarly.

LeMMA 2. Suppose 3 sequences of scalars $f_{n}, g_{n 1}, \cdots, g_{n p}$ and sequences of random variables $U_{n 1}, U_{n 2}, \cdots, U_{n p}$ such that $\left(U_{n 1}, U_{n 2}\right.$, $\left.\cdots, U_{n p}\right) \stackrel{w}{\rightarrow}$ nondegenerate $\left(U_{1}, U_{2}, \cdots, U_{p}\right)$ and $f_{n}+\sum_{i=1}^{p} g_{n i}$ $U_{n i} \stackrel{\omega}{\rightarrow}$ nondegenerate $W_{p}$. Then $\lim \sup \left|f_{n}\right|<\infty, \lim \sup \left|g_{n i}\right|<\infty$, $i=1,2, \cdots, p$.

Proof. Note that if $\lim \sup \left|g_{n i}\right|<\infty, i=1,2, \cdots, p$, then $\lim \sup \left|f_{n}\right|<\infty$, for otherwise, $\exists$ a subsequence $\left\{n_{k}\right\}$ such that $f_{n_{k}} \rightarrow \pm \infty$ and $g_{n_{k} i} \rightarrow g_{i}$. It follows from a theorem of Prokhorov [4, Theorem 1.10, p. 166] that $\mathcal{L}\left(\sum_{i=1}^{p} g_{n_{k^{i}}} U_{n_{k^{i}}}\right) \rightarrow \mathcal{L}\left(\sum_{i=1}^{p} g_{i} U_{i}\right)$ and from lemma $1(\mathrm{~b})$ that $\mathcal{L}\left(f_{n_{k}}+\sum_{i=1}^{p} g_{n_{k} i} U_{n_{k} i}\right) \rightarrow$ a defective probability distribution which cannot be $\mathcal{L}\left(W_{p}\right)$. We shall show that $\lim \sup \left|g_{n i}\right|<\infty, i=1,2, \cdots, p$ by induction on $p$. Suppose first that $p=1$ and $\lim \sup \left|g_{n 1}\right|=\infty$. Then $\exists$ a subsequence $\left\{n_{k}\right\}$ such that $\left|g_{n_{k} 1}\right| \rightarrow \infty$, and from lemma $1(\mathrm{a}) f_{n_{k}} / g_{n_{k} 1}+U_{n_{k} 1} \stackrel{p}{\rightarrow} 0$. This contradicts $\mathcal{L}\left(U_{n_{k} 1}\right) \rightarrow \mathcal{L}\left(U_{1}\right)$ from lemma $1(\mathrm{~b})$ if $f_{n_{k}} / g_{n_{k} 1}$ is unbounded. If $f_{n_{k}} \lg _{n_{k} 1}$ is bounded, then $\exists$ a subsequence $n_{k}$ ' such that $f_{n_{k}} / g_{n_{k^{\prime}}{ }^{\prime} w_{w}}$ converges to some real $c$, and then, $\mathcal{L}\left(f_{n_{k^{\prime}}} / g_{n_{k^{\prime}}{ }^{\prime}}+\right.$ $\left.U_{n_{k^{\prime}} 1}\right) \stackrel{w}{\rightarrow} \mathcal{L}\left(U_{1}+c\right)$ nondegenerate, which contradicts $f_{n_{k^{\prime}}} / g_{n_{k^{\prime} 1}}+$ $U_{n^{\prime}{ }^{\prime}+} \stackrel{p}{\rightarrow} 0$. Now suppose the result is true for $p$, and $\mathcal{L}\left(f_{n}+\right.$ $\left.\sum_{i=1}^{p+1} g_{n i} U_{n i}\right) \rightarrow$ nondegenerate $\mathcal{L}\left(W_{p+1}\right)$. Suppose lim sup $\left|g_{n i_{0}}\right|=\infty$. Then $\exists$ a subsequence $\left\{n_{k}\right\}$ such that

$$
\frac{f_{n_{k}}}{g_{n_{k} i_{0}}}+\sum_{i \neq i_{0}} \frac{g_{n_{k} i}}{g_{n_{k} i_{0}}} U_{n_{k} i}+U_{n_{k} i_{0}} \stackrel{P}{\rightarrow} 0
$$

and

$$
\mathcal{L}\left(\frac{f_{n_{k}}}{g_{n_{k} i_{0}}}+\sum_{i \neq i_{0}} \frac{g_{n_{k} i}}{g_{n_{k} i_{0}}} U_{n_{k} i}\right) \rightarrow \text { nondegenerate } \mathcal{L}\left(-U_{i_{0}}\right) .
$$

It follows from the induction hypothesis that $\exists\left\{n_{\ell}\right\} \subset\left\{n_{k}\right\}$ such that

$$
\mathcal{L}\left(\frac{f_{n_{\ell}}}{g_{n_{\ell} i_{0}}}+\sum_{i \neq i_{0}} \frac{g_{n_{\ell} i}}{g_{n_{\ell} i_{0}}} U_{n_{\ell} i}+U_{n_{\ell} i_{0}}\right) \rightarrow \mathcal{L}\left(\alpha+\sum_{i \neq i_{0}} \beta_{i} U_{i}+U_{i_{0}}\right)
$$

with $\alpha$ and $\beta_{i}$ finite. Hence $P\left(\sum_{i \neq i_{0}} \beta_{i} U_{i}+U_{i_{0}}+\alpha=0\right)=1$, which 
contradicts $\left(U_{1}, U_{2}, \cdots, U_{p+1}\right)$ is nondegenerate. Thus lim sup $\left|g_{n i}\right|$ $<\infty, i=1,2, \cdots,(p+1)$, and the proof is complete.

THEOREM. Let $U_{n}{ }^{\prime}=\left(U_{n 1}, U_{n 2}, \cdots, U_{n p}\right), V_{n}{ }^{\prime}=\left(V_{n 1}, V_{n 2}, \cdots, V_{n p}\right)$, $U^{\prime}=\left(U_{1}, U_{2}, \cdots, U_{p}\right)$, and $V^{\prime}=\left(V_{1}, V_{2}, \cdots, V_{p}\right)$. If $\mathcal{L}\left(U_{n}\right) \rightarrow$ nondegenerate $\mathcal{L}(\boldsymbol{U})$ and $\mathcal{L}\left(\boldsymbol{V}_{n}\right) \rightarrow$ nondegenerate $\mathcal{L}(\boldsymbol{V})$ and $U_{n} \sim V_{n}$ for sufficiently large $n$, then $U \sim V$.

Proof. Let $\mathcal{L}\left(\boldsymbol{V}_{n}\right)=\mathcal{L}\left(\boldsymbol{A}_{n}+\boldsymbol{B}_{n} \boldsymbol{U}_{n}\right)$ for $n \geqq N$ with $\boldsymbol{A}_{n}{ }^{\prime}=\left(a_{n 1}, a_{n 2}\right.$, $\left.\cdots, a_{n p}\right)$ and $\boldsymbol{B}_{n}=\left(b_{n i j}\right)$. It follows from the Mann-Wald theorem [2, Theorem 5, p. 223] that $\mathcal{L}\left(U_{n 1}\right)=\mathcal{L}\left(a_{n 1}+\sum_{j=1}^{p} b_{n 1 j} U_{n j}\right) \rightarrow$ nondegenerate $\mathcal{L}\left(\boldsymbol{U}_{1}\right)$ and from lemma 2 that lim sup $\left|a_{n 1}\right|<\infty$, $\lim \sup \left|b_{n 1 j}\right|<\infty, j=1,2, \cdots, p$. Similarly $\lim \sup \left|a_{n i}\right|<\infty$, $\lim \sup \left|b_{n i j}\right|<\infty, i=1,2, \cdots, p ; j=1,2, \cdots, p$. Hence $\exists\left\{n_{k}\right\}$ such that $\boldsymbol{A}_{n_{k}} \rightarrow \boldsymbol{A}$ and $\boldsymbol{B}_{n_{k}} \rightarrow \boldsymbol{B}$ with elements of $\boldsymbol{A}$ and $\boldsymbol{B}$ finite. Since $\mathcal{L}\left(\boldsymbol{V}_{n_{k}}\right)=\mathcal{L}\left(\boldsymbol{A}_{n_{k}}+\boldsymbol{B}_{n_{k}} \boldsymbol{U}_{n_{k}}\right)$ for sufficiently large values of $n_{k}$ it follows from the theorem of Prokhorov referred to earlier that $\mathcal{L}\left(\boldsymbol{V}_{n_{k}}\right) \rightarrow \mathcal{L}(\boldsymbol{A}+\boldsymbol{B} \boldsymbol{U})$. Since $\mathcal{L}\left(\boldsymbol{V}_{n_{k}}\right)$ also converges to $\mathcal{L}(\boldsymbol{V})$ it follows that $\mathcal{L}(\boldsymbol{V})=\mathcal{L}(\boldsymbol{A}+\boldsymbol{B U})$. Since $\boldsymbol{V}$ and $\boldsymbol{U}$ are both nondegenerate, $\boldsymbol{B}$ is nonsingular, and the proof is complete.

The following analogous result for strong similarity is proved in [5]. If $\mathcal{L}\left(U_{n}\right) \rightarrow$ nondegenerate $\mathcal{L}(U), \quad \mathcal{L}\left(V_{n}\right) \rightarrow$ nondegenerate $\mathcal{L}(V)$, and $U_{n} \stackrel{s}{\sim} V_{n}$ for sufficiently large $n$, then $U \stackrel{s}{\sim} V$. One can also define an intermediate type of similarity with diagonal matrices $B$, and the theorem remains true for this type of similarity also.

It is easy to see that any two (nondegenerate) multivariate normal distributions are similar, and it follows from the above theorem that if $\mathcal{L}\left(\boldsymbol{U}_{n}\right) \rightarrow \mathcal{N}(\boldsymbol{\mu}, \Sigma)$, then $\mathcal{L}\left(\boldsymbol{A}_{n}+\boldsymbol{B}_{n} U_{n}\right) \rightarrow \mathcal{N}(\nu, \psi)$ if $\boldsymbol{B}_{n}$ are nonsingular (for sufficiently large values of $n$ ) and the limit law of $A_{n}+$ $B_{n} U_{n}$ exists and is nondegenerate. This justifies the usage that a sequence of random vectors $U_{n}$ is asymptotically multivariate normal without specifying the "norming constants" $\boldsymbol{A}_{n}$ and $\boldsymbol{B}_{n}$.

\section{REFERENCES}

1. B. V. Gnedenko and A. N. Kolmogorov, Limit distributions for sums of independent random variables. Addison-Wesley Publishing Company, Inc., 1954.

2. H. B. Mann and A. Wald, On stochastic limit and order relations. Ann. Math. Statist. 14 (1943), 217-226.

3. R. Ponnapalli, Types of laws of random vectors and best estimators. Journal of Multivariate Analysis 5 (1975), 278-281.

4. Yu. V. Prokhorov, Convergence of random processes and limit theorems in probability theory. Theor. Prob. Appl. 1 (1956), 157-214.

5. K. Takano, On the many-dimensional distribution functions. Ann. Inst. Stat. Math., Tokyo 5 (1954), 40-58.

University of Alberta, Edmonton, Alberta, Canada 